\title{
Usefulness of P16 and KI67 Immunostaining in Cervical Smear Cytology in Guatemalan Women: A Cross-Sectional Study
}

\author{
Alberto García, MD, $P h D^{1,8}$, Angel Velarde MD, MSCE ${ }^{2 *}$, Francisco Quereda, MD, \\ $P h D^{3}$, Jaume Ordi, MD, $P_{h} D^{4}$, Orlando Rodas, $M D, P h D^{5}$, Erick Soch, $M D^{6}, H$. Arturo \\ Morales, $\mathrm{MD}^{6}$, Federico $\mathrm{Nave}^{7}$ and Carlos López, MD
}

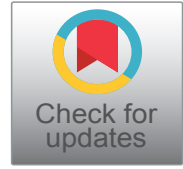

${ }^{1}$ Colposcopy Unit, Instituto de Cancerología y Hospital Dr. Bernardo del Valle S, Guatemala

${ }^{2}$ Research Director of Liga Nacional Contra El Cáncer e Instituto de Cancerología y Hospital Dr. Bernardo del Valle S, Guatemala

${ }^{3}$ Department of Gynecology of the hospital San Joan Alicante and Professor of the University Miguel Hernández de Elche, Spain ${ }^{4}$ Department of Pathology, Clinic of the hospital of Barcelona, Spain

${ }^{5}$ Department of Molecular Pathology, Faculty of medicine of the University of San Carlos de Guatemala, Guatemala

${ }^{6}$ Pathology Department, Instituto de Cancerología y Hospital Dr. Bernardo del Valle S, Guatemala

${ }^{7}$ Research Director of the University of San Carlos de Guatemala

${ }^{8}$ Professor of Biology and researcher, Faculty of medicine of the University of San Carlos de Guatemala

*Corresponding author: Angel Velarde, Research Director of Liga Nacional Contra El Cáncer e Instituto de Cancerología y Hospital Dr. Bernardo del Valle S $6^{a}$ Avenenida 6-58, Z.11, Guatemala

\begin{abstract}
Background: Conventional cytology is an economic method for screening abnormalities in cervical smears, but with implications in false positive and false negative as compared to better detection of transformed cells with the Dual staining P16/KI67 proteins in cervical smears, therefore, we evaluate the validity and reproducibility of cytology with double staining of P16 and KI67 as compared to conventional cytology in cervical smears.

Patients and methods: Participants were 210 Guatemalan women with abnormalities in conventional cytology (ASCUS, AGUS, ASCH, LSIL, HSIL, and cervical cancer) that consulted from 2013-2014 to the colposcopy Unit of Liga Nacional Contra El Cáncer e Instituto de Cancerología y Hospital Dr. Bernardo del Valle S. in Guatemala, from whom smear cytology was processed with P16 and KI67 immunostaining, and histological sections with P16 immunostaining as gold standard. They were evaluated by three blinded pathologists and one independent cytotechnologist.

Results: The sensitivity and specificity of Dual staining P16/ KI67 for detecting abnormalities in cervical smears was $78.95 \%, 95 \%$ confidence interval $(\mathrm{Cl}) 71.03-86.87$ and
\end{abstract}

$94.74 \%, 95 \% \mathrm{Cl} 89.06-100$ respectively, as compare to conventional cytology in which it was $78.64 \%$ and $75.85 \%$, respectively; positive predictive value (PPV) and negative predictive value (NPV) of Dual staining $\mathrm{P} 16 / \mathrm{KI} 67$ were $95.74 \%$, $95 \% \mathrm{Cl} 91.13-100.0$ and $75 \%, 95 \% \mathrm{Cl} 65.82-84.18$, as compare to conventional cytology in which it was $79.41 \%$ and $75 \%$ respectively. In women older than 30 years the sensitivity and PPV of Dual staining P16/KI67 was $78.43 \%$ and $95.24 \%$, as compare in women less than 30 years in which it was $41.67 \%$ and $83.33 \%$ respectively. Interobserver agreement weighted Kappa indices for cervical smear cytology with double staining ranged between 0.66 to 0.83 .

Conclusion: Dual staining P16/KI67 in women older than 30 years showed better sensitivity and PPV values, suggesting greater utility in this age group, although the sensitivity was like conventional cytology, but with greater specificity and PPV.

\section{Keywords}

Immunostaining, P16, KI67, Cervical intraepithelial neoplasia, Cervical smear cytology, Women, Guatemala

Citation: García A, Velarde A, Quereda F, Ordi J, Rodas O, et al. (2019) Usefulness of P16 and KI67 Immunostaining in Cervical Smear Cytology in Guatemalan Women: A Cross-Sectional Study. Int J Oncol Res 2:019. doi.org/10.23937/2643-4563/1710019

Accepted: October 16, 2019; Published: October 18, 2019

Copyright: (c) 2019 García A, et al. This is an open-access article distributed under the terms of the Creative Commons Attribution License, which permits unrestricted use, distribution, and reproduction in any medium, provided the original author and source are credited. 


\section{Introduction}

Globally, including in Latin America, low- and middle-income countries (LMICs) are experiencing an epidemiologic transition from infectious diseases to cancer and chronic diseases, and in Latin America cancer is the second leading cause of death $[1,2]$.

Cervical cancer ranks second in incidence and mortality behind breast cancer in lower human development index setting, with an estimated 570,000 cases and 311,000 deaths in 2018 worldwide [3]. In Latin America Cervical cancer remains the number one cause of mortality due to malignant neoplasm among 20 to 40 -year-old women [4]. Virtually all cervical cancers $(99 \%)$ are liked to genital infection with human papilloma viruses (HPV), and persistent genital HPV infection causes cervical cancer in women. A large majority (around $85 \%$ ) of the global burden occurs in the less developed regions, where it accounts for almost $12 \%$ of all female cancers [5].

In Guatemalan general population (GP) and among a group of female sex workers (SW) the HPV prevalence estimates were $38.1 \%(95 \% \mathrm{Cl}=32.5-43.8)$ and $67.3 \%$ $(95 \% \mathrm{Cl}=61.7-72.6)$ [6]. It is well known that high risk $\mathrm{HPV}$ is responsible for causing preinvasive lesions of the cervix in $36 \%, 63 \%$ and $80 \%$ of the cervical intraepithelial neoplasia (CIN)I, II and III [7].

In Guatemala, by 2018, the age-standardized incidence rate of cervical cancer was 21.1, and the mortality 11.7 per 100,000 , ranked as the third cancer, and fourth as a cause of death [8], and remains as a major public health problem [9].

The sensitivity and specificity of pap smear has being reported as $57 \%$ and $76 \%$, and for colposcopy $92 \%$ and $67 \%$ respectively in detecting premalignant and malignant cervical lesion [10].

It has been described the capacity of detecting cells that are in the process of transforming to neoplasia with Dual stain testing P16 and KI67, which is a useful surrogate biomarker of cervical neoplasia with higher specificity and increases the precision of conventional cytology. The Sensitivity of Dual-stain testing for the detection of biopsy-confirmed CIN2+ during preliminary follow-up within the group of Pap negative/HPV positive women was $91.9 \%$ for $\mathrm{CIN2+}$ (34/37 cases), and $96.4 \%$ for CIN3+ (27/28 cases). Specificity was $82.1 \%$ for CIN2+ on biopsy, and $76.9 \%$ for CIN3+, respectively [11-13].

A variety of studies have shown a sensitivity of $93.43 \%$ and specificity of $78.96 \%$ which is higher when compare to women younger than 30 years for detecting cervical neoplasia using the Dual stain testing P16 and $\mathrm{KI} 67$, and the diagnostic accuracy improved when both the stains were used in conjunction, of note, there were no studies in which the Dual stain has being done using cervical smears, but only in liquid base [11,13-16]. P16 and Ki-67 have emerged as important biomarkers for the detection of high-risk human papilloma virus and in confirming the histopathological diagnosis [17]. Therefore, we aim to evaluate the validity and reproducibility of cytology with double staining of P16 and KI67 in cervical smears.

\section{Patients and Methods}

\section{Study design}

A cross sectional study from 2013 to 2014 of 210 Guatemalan women referred to the colposcopy unit of Instituto de Cancerología y Hospital Dr. Bernardo del Valle S. in Guatemala.

\section{Inclusion criteria}

Women referred with abnormal conventional cytology as Atypical squamous cells of undetermined significance (ASCUS), atypical glandular cells of undetermined significance (AGUS), Atypical squamous cells (ASCH), low-grade squamous intraepithelial lesion (LSIL), highgrade squamous intraepithelial lesion (HSIL), and cervical cancer (CC).

\section{Exclusion criteria}

Patients treated by any oncological disease of the cervix, as well as any pregnant woman.

\section{Sample size calculation}

With a confidence level of $97 \%$, to be able to detect a difference of $5 \%$, we were interested in PPV (probability that the disease is present when the test is positive) of the Dual staining P16/KI67 and we wanted it to be $90 \%$, considering $12 \%$ of missing data, the total sample size required was 190 participants.

\section{Patient management}

For each patient, new samples of cervical smear for cytology were obtained as part of their routine standard of care, another for histology that included cervical biopsies and in 45 cases cervical conization tissue. According to the consensus of the evaluating doctors, of the 210 samples, 20 were excluded from the study due to poor quality for interpretation. In total there were 190 cases, each patient read and signed the informed consent, all required permission were obtained.

\section{Procedural step process}

Prior to the colposcopy examination, a sample of cervical and endocervical exfoliation was obtained with Ayre's plastic palettes and cytobrush brushes, placing the rub on lamellae with a load and preserved with buffered alcohol. Subsequently, the evaluation was carried out with aleisegang optik1-02 brand colposcope, the cervix was previously prepared with $5 \%$ acetic acid; documenting the findings, capturing the images and completing the institutional card of colposcopy, in which the observed findings are placed, number of biopsies obtained and colposcopic impression. Obtaining samples of the cervix by means 
of a Kevorkian biopsy forceps and in cases where no transformation zone was observed, curetting was performed with endocervical legra.

\section{Sample processing}

The processing of both cytological and histological samples was carried out in the molecular pathology laboratory of the Biomedical Research Center of the Faculty of Medicine of the University of San Carlos de Guatemala. All step process of sample packaging and transportation where consider to be appropriate from the hospital to the laboratory which is located about three blocks from the hospital.

For the cytologies, Dual staining P16/KI67 test was used following the manufacturer's instructions (Laboratories $\mathrm{mtm}$ Roche from Germany); subsequently they are stained with hematoxylin and Eosin.

For cervical biopsies and cones, $4 \mu \mathrm{m}$ thick cuts were made for immunostaining of P16, using the in Vision (Dako), P16INK4a system was detected using the CINtec histology kit (clone E6H4, mtm Laboratories, Heidelberg, Germany), following the manufacturer's instructions. Histological sections for both P16 Immunostaining and for staining hematoxylin and Eosin were obtained from the same paraffin block.

Each cytology was previously evaluated by a cytotechnologist with more than 15 years of experience, dictating the quality of the rub. Subsequently, each of the three independent pathologists ruled the cytomorphological findings, as well as whether the double staining test was positive or not, when observing in the same cell red nucleus and brown cytoplasm.

In the case of histology samples, histomorphology findings were ruled, as well as whether P16 staining was positive or not, being positive if the tissue is stained with diffuse brown color.

\section{Statistical analysis}

A 2 times 2 table was established to calculate the sensitivity and specificity of both the conventional cytology and the Dual P16 and KI67 staining test in less than 30 years and older than 30 years, as well as PPV and NPV with its $95 \%$ confidence interval. Kappa coefficient was calculated between pathologist from Guatemala and Spain using Epidat version 3.0.

\section{Samples interpretation}

Each cytology was previously evaluated by a cytotechnologist with more than 15 years of experience. Subsequently, each of the three independent pathologists ruled the cytomorphological findings. Each pathologist and cytotechnologist report their findings with the Bethesda system of the year 2001, independently and blindly as reported by the others.

\section{Results}

Of the 210 participating patients, 20 were excluded due to poor sample quality. Of the 190 patients, the mean age and standard deviation was $41 \pm 13.9$ years. The most numerous age range was between 26 to 35 years $(29.5 \%)$, followed by the range 36 to 45 years (26.8\%). According to previous pregnancies, 43 patients (24\%) have 3 children, followed by 33 patients (18.75\%) with 1 child and 32 patients (18.18\%) with more than 6 children.

Table 1: Demographic characteristics of patients.

\begin{tabular}{|c|c|c|}
\hline Age & N (190) & $\%$ \\
\hline$<20$ & 7 & 3.7 \\
\hline 21 a 25 & 16 & 8.4 \\
\hline 26 a 35 & 56 & 29.5 \\
\hline 36 a 45 & 51 & 26.8 \\
\hline 46 a 55 & 29 & 15.3 \\
\hline 56 a 65 & 16 & 8.4 \\
\hline$>66$ & 15 & 7.9 \\
\hline Pregnancias & $\mathbf{n}$ & $\%$ \\
\hline 0 & 6 & 3.2 \\
\hline 1 & 34 & 17.9 \\
\hline 2 & 29 & 15.3 \\
\hline 3 & 48 & 25.3 \\
\hline 4 & 24 & 12.6 \\
\hline 5 & 13 & 6.8 \\
\hline$>6$ & 36 & 18.9 \\
\hline Contracpetive methods & $\mathbf{n}$ & $\%$ \\
\hline None & 123 & 64.7 \\
\hline Pomerooy or rings & 42 & 22.1 \\
\hline Oral contraceptives & 12 & 6.3 \\
\hline Quaterly & 8 & 4.2 \\
\hline Jadell & 3 & 1.6 \\
\hline Intrauterine device & 2 & 1.1 \\
\hline
\end{tabular}

Table 2: Cervical cytologies according to conventional and Dual P16/KI67 staining.

\begin{tabular}{|l|l|l|}
\hline & Conventional n (\%) & P16 and KI67 n (\%) \\
\hline Negative & $0(0)$ & $65(34.21)$ \\
\hline${ }^{a}$ AGUS & $4(2.1)$ & $1(0.53)$ \\
\hline${ }^{b}$ ASCUS & $27(14.21)$ & $4(2.11)$ \\
\hline 'ASCH & $3(1.58)$ & $2(1.05)$ \\
\hline d LSIL & $54(28.42)$ & $24(12.63)$ \\
\hline eHSIL & $75(39.47)$ & $72(37.89)$ \\
\hline fISC & $22(11.58)$ & $14(7.37)$ \\
\hline gIAC & $5(2.63)$ & $8(4.21)$ \\
\hline
\end{tabular}

aAGUS: Atypical Glandular Cells Of Undetermined Significance; ${ }^{b}$ ASCUS: Atypical Squamous Cell Of Undetermined Significance; ${ }^{\mathrm{A}} \mathrm{ASCH}$ : Atypical Squamous Cell Of Undetermined Significance; 'LSIL: Low-Grade Squamous Intraepitheli-

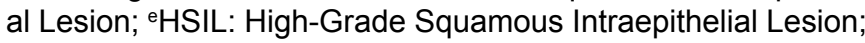
fISC: Invasive Squamous Carcinomas; 9IAC: Invasive Adenocarcinoma. 
To the question of the use of contraceptive methods used, 123 patients (64.7\%) report not having used any, the most commonly used method was Pomeroy or rings, 42 patients $(22.1 \%)$, see Table 1 . Table 2 shows the distribution of conventional cytology versus Dual staining P16/ KI67, and Table 3 shows the distribution according to histology diagnosis with P16 staining.

From each patient, a cytology sample with double staining P16 and KI67 and one or two histology (biopsy and cervical cone) with P16 staining were evaluated. There were 4 evaluators; 3 pathologists of which one was from Spain (A) and two from Guatemala (B and C); and a cytotechnologist (D). The concordance index was obtained with Kappa weighted with $95 \% \mathrm{Cl}$. It is observed that the index for cytology is between 0.66 to 0.83 , being significant with $p<0.0001$, being the largest agreement between the expert pathologist and the cytotechnologist. In the biopsy samples they obtained a better concordance index, which was between 0.80 and 0.89 , considered very well with $p<0.0001$. With respect to the interpretation of cervical cones, there was a concordance index between 0.76 and 0.90 , respectively

Table 3: Cytology with Dual P16/Ki67, according to histology diagnosis with P16 staining.

\begin{tabular}{|l|l|l|l|l|l|}
\hline & & \multicolumn{3}{|l|}{ Morphology in cytology with immunostaining P16/Ki67 } \\
\hline $\begin{array}{l}\text { Final diagnosis, staining } \\
\text { P16 }\end{array}$ & No. cases & Negative No. (\%) & ASC-US, LSIL× No. (\%) & HSIL No. (\%) & CC ${ }^{\text {h No. (\%) }}$ \\
\hline Negative & 53 & $45(84.90)$ & $4(7.55)$ & $4(7.55)$ & $0(0)$ \\
\hline LSIL & 23 & $8(34.78)$ & $15(65.22)$ & $0(0)$ & $0(0)$ \\
\hline HSIL & 78 & $12(15.38)$ & $11(14.10)$ & $52(66.67)$ & $3(3.85)$ \\
\hline Carcinomay & 36 & $0(0)$ & $1(2.78)$ & $16(44.44)$ & $19(52.78)$ \\
\hline
\end{tabular}

xIncludes 1 case of AGUS, 4 of ASCUS, 2 of ASCH and 24 of LSIL; yIncludes 6 cases of Invasive adenocarcinoma; aAGUS: Atypical Glandular Cells Of Undetermined Significance; ${ }^{b}$ ASCUS: Atypical Squamous Cell Of Undetermined Significance; ${ }^{\circ} A S C H$ :

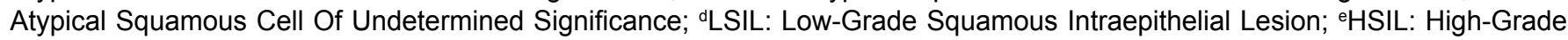
Squamous Intraepithelial Lesion; ' ISC: Invasive Squamous Carcinomas; ${ }^{h}$ CC: Cervical Cancer.

Table 4: Interobserver agreement.

\section{Type of sample interpreted}

\begin{tabular}{|c|c|c|c|c|c|c|}
\hline & \multicolumn{2}{|c|}{ Cervical cytology, double staining P16/Ki67 } & \multicolumn{2}{|c|}{ Cervical biopsy, staining P16 } & \multicolumn{2}{|c|}{ Cervical cone, staining with P16 } \\
\hline & Kappa indexa & $95 \% \mathrm{Cl}$ & Kappa index ${ }^{a}$ & $95 \% \mathrm{Cl}$ & Kappa index ${ }^{a}$ & $95 \% \mathrm{Cl}$ \\
\hline A-B & $0.77^{*}$ & $0.69-0.84$ & $0.83^{*}$ & $0.77-0.89$ & $0.81^{*}$ & $0.60-1.0$ \\
\hline$A-C$ & $0.74^{*}$ & $0.65-0.84$ & $0.89^{*}$ & $0.85-0.93$ & $0.90^{*}$ & $0.81-0.99$ \\
\hline A-D & $0.83^{*}$ & $0.77-0.89$ & ---- & ----- & ----- & ----- \\
\hline$B-C$ & $0.66^{*}$ & $0.57-0.75$ & $0.80^{*}$ & $0.74-0.87$ & $0.76^{*}$ & $0.53-0.98$ \\
\hline$B-D$ & $0.75^{\star}$ & $0.66-0.81$ & ---- & ----- & ----- & ----- \\
\hline C-D & $0.75^{*}$ & $0.67-0.84$ & ---- & ----- & ----- & ---- \\
\hline
\end{tabular}

A: Expert Pathologist; B y C: Guatemalan Pathologists; D: Cytotechnologist; K: Weighted Kappa; Cl: Confidence Interval; "P value $<0.0001$.

Table 5: Results of histology interpretation with H\&E and with P16.

\begin{tabular}{|c|c|c|c|c|}
\hline & \multicolumn{2}{|c|}{ Cervical bioposies } & \multicolumn{2}{|l|}{ Cervical cones } \\
\hline & $\begin{array}{l}\text { H\&E staining } \\
\text { n (\%) }\end{array}$ & P16 n (\%) & $\begin{array}{l}\text { H\&E staining } \\
\text { n (\%) }\end{array}$ & P16 n (\%) \\
\hline Negative & $53(27.9)$ & $53(27.9)$ & $4(8.9)$ & $4(8.9)$ \\
\hline dLSIL & $34(17.9)$ & $23(12.1)$ & $4(8.9)$ & $0(0)$ \\
\hline eHSIL & $68(35.9)$ & $78(41.1)$ & $31(68.9)$ & $35(77.8)$ \\
\hline \multicolumn{5}{|l|}{ fISC } \\
\hline Microinvasive & $6(3.1)$ & $3(1.6)$ & $3(6.7)$ & $1(2.2)$ \\
\hline Invasive & $23(12.1)$ & $26(13.7)$ & $3(6.7)$ & $5(11.1)$ \\
\hline Invasive adenocarcinoma & $6(3.1)$ & $7(3.7)$ & $0(0)$ & $0(0)$ \\
\hline Total & $190(100)$ & $190(100)$ & $45(100)$ & $45(100)$ \\
\hline
\end{tabular}

dLSIL: low-grade squamous intraepithelial lesion; eHSIL: high-grade squamous intraepithelial lesion; fISC: Invasive squamous carcinomas. 
being considered good to very good with $p<0.0001$, see Table 4.

The distribution of the cytomorphological interpretation of the gold standard that was the diagnosis by biopsy or cone with the corresponding staining. Because in some cases there was a discrepancy between cytology and biopsy, specifically cases in which cytology reported HSIL or invasive cancer and in biopsy reported cervicitis or LSIL, diagnostic cones were performed in some cases. Therefore, it was considered in these cases the final or conclusive diagnosis is the result of the cervical cone (Table 5).

For the Dual staining P16/KI67, it is observed that for both types of cytologies, conventional and double staining with P16/KI67, the sensitivity is similar 78.64 and $78.95 \%$ respectively. However, the specificity was different being 75.85 and $94.74 \%$ respectively, higher for double staining because there are fewer false positive cases (Table 6).

With respect to PPV, that is, the percentage of probability that the positive test is positive in the patient who is ill (diagnosis $\geq \mathrm{HSIL}$ ) was higher in cytology with double staining 95.74\% with 95\% $\mathrm{Cl}$ 91.13-100.0. The PPV of conventional cytology and colposcopy was 79.41. The NPV, that is the percentage or probability that the test being negative, the patient is healthy, was similar in both types of cytologies with $75 \%$ in each one. The sensitivity, specificity, PPV and NPV of double staining in cytology were compared, according to two age groups, in < 30 and older $>30$ years. The confirmatory test was the biopsy or cervical cone with P16 staining. He evidenced that the highest sensitivity and PPV of the test is in the group > 30-years-old, 78.43 and $95.24 \%$ respectively. Specificity and NPV were slightly higher in the $<30$ years old group: 95.83 and $83.33 \%$ (Table 7 ).

\section{Discussion}

When comparing the interpretation of the two types of cytology, (conventional staining or pap and double staining P16/KI67), there were differences especially in the cytological diagnoses of ASCUS and LSIL.

In the cases less than HSIL, the results between cytology with conventional staining and double stain- ing were different: ASCUS 27/190 (14.21\%) and 4/190 (2.11\%); LSIL 54/190 (28.42\%) and 24/190 (12.63\%) respectively, therefore the difference for ASCUS of $12 \%$ and LSIL of $16 \%$. The use of this screening technique would have prevented a $25 \%$ extension of the study because it was low grade, with which initial follow-up, or eventually colposcopy, could be performed to determine the next step.

For lesions of HSIL there was a $4 \%$ overall difference between both types of stains, which could suggest that it detects fewer cases (less sensitivity) or as histological correlation seems to show, which has less false positives.

The interobserver reproducibility of good concordance, demonstrates a simplification and greater coherence and safety in the diagnosis. In this sense, the interobserver reproducibility of P16/KI67 staining in cervical smear cytology is reflected in the concordance index with weighted Kappa that was 0.66 to 0.83 (qualifiable as good); in a similar but liquid based study Wentzensen, et al. reported that it was from 0.65 to 0.81 [18], like what we found.

In histology, P16 staining was evaluated with respect to the interobserver concordance index or agreement between pathologists. In this study it was found that the weighted Kappa index of three pathologists for histomorphological interpretation of biopsies and cervical cones with P16 staining was between 0.83-0.89 and $0.76-0.90$ respectively; this concordance is considered good to very good, with significance ( $p<0.0001)$. In a similar study the Concordance Index was evaluated giving an average of 0.89 for biopsies and cervical cones [19].

Regarding the results of cones, with H\&E and P16 staining, it is observed that in 3 cones with P16 they are reclassified from microinvasive to invasive. This may be relevant in influencing the surgical management of the patient for definitive treatment.

The present study reports a sensitivity to detect cytomorphological changes corresponding to lesion of HSIL with conventional staining and with double staining, 78.64 and $78.95 \%$ respectively. Therefore, the sensitivity value in both cytologies was similar.

In a meta-analysis which reviewed 24 articles, the

Table 6: Sensitivity, specificity, PPV and NPV of conventional cytology staining versus staining with P16/Ki67.

\begin{tabular}{|l|l|l|l|l|}
\hline Test method & Sensitivity, $\mathbf{9 5} \mathbf{c} \mathbf{C l}$ & Specificity, 95\%Cl & PPV, 95\%Cl & NPV, 95\%Cl \\
\hline Conventional & $78.64,70.24-87.04$ & $75.86,66.30-85.43$ & $79.41,71.07-87.75$ & $75.00,65.38-84.62$ \\
\hline P16/Ki67 staining & $78.95,71.03-86.87$ & $94.74,89.06-100.0$ & $95.74,91.13-100.0$ & $75.00,65.82-84.18$ \\
\hline
\end{tabular}

Table 7: Sensitivity, specificity, PPV, NPV of P16/Ki67 by age group.

\begin{tabular}{|l|l|l|l|l|}
\hline & Sensitivity, (95\%Cl) & Specificity, (95\%Cl) & VPP, (95\%CI) & NPV, (95\%CI) \\
\hline$<30$ años & $41.67,(9.61-73.73)$ & $95.83,(85.76-100)$ & $83.33,(45.18-100)$ & $76.67,(59.87-93.47)$ \\
\hline$>30$ años & $78.43,(69.96-86.90)$ & $92.31,(69.96-86.90)$ & $95.24,(90.09-100)$ & $68.57,(56.98-80.16)$ \\
\hline
\end{tabular}


sensitivity of conventional cytologies was $65 \%$. This suggests that in our environment the sensitivity is higher, however, we must consider that our study was conducted in patients referred to the Colposcopy Unit with cytology results greater than or equal to ASCUS [20]. Therefore, to detect lesions of HSIL (which is clinically relevant) the sensitivity of dual staining was similar to conventional, but dual staining P16/KI67 has a greater PVV with specificity of $94.74 \%$ versus $75.86 \%$ of the cytology with conventional staining.

Comparable studies, such as that of Murphy, et al. [21], report a similar specificity, having a value of $95.7 \%$, and report that the false positive rate for conventional cytology is $30 \%$. The latter is somewhat higher than ours, but again we must consider that our sample had more than $50 \%$ of women with a lesion of HSIL.

Two age groups were also analyzed, depending on whether they were under or over 30-years-old. Double staining in less than 30 years presented a sensitivity and specificity of $41.67 \%$ and $95.83 \%$ respectively, however, in the group above 30 years the sensitivity and specificity was $78.43 \%$ and $95.24 \%$ respectively.

These data are comparable with those of a study conducted by Jaume Ordi, et al. [13] which showed that in patients younger than 30 years the sensitivity and specificity was $78 \%$ and $91 \%$ respectively, and in over 30 years the sensitivity and specificity was 86.5 and $94.8 \%$ respectively. The specificity is similar in our study and sensitivity is likely to be more limited because the number of cases with lesions greater than HSIL was much lower among women who referred us at a younger age.

\section{Conclusion}

Dual staining P16/KI67 in women older than 30 years showed better sensitivity and PPV values, suggesting greater utility in this age group, although the sensitivity was like conventional cytology, but with greater specificity and PPV.

\section{Acknowledgement}

The authors thank to the National Science and Technology Fund of Guatemala SENACYT-FODECYT for supporting this study.

\section{Conflict of Interest}

None to declare

\section{Research Funding}

SENACYT-FODECYT, National Science and Technology Fund of Guatemala.

\section{References}

1. Frech $S$, Muha CA, Stevens LM, Trimble EL, Brew R, et al. (2018) Perspectives on Strengthening Cancer Research and Control in Latin America Through Partnerships and Diplomacy: Experience of the National Cancer Institute's Center for Global Health. J Glob Oncol 22: 1-11.
2. Torres Á, Oliver J, Frecha C, Montealegre AL, Quezada-Urbán R, et al. (2017) Cancer Genomic Resources and Present Needs in the Latin American Region. Public Health Genomics 20: 194-201.

3. Bray F, Ferlay J, Soerjomataram I, Siegel RL, Torre LA, et al. (2018) Global cancer statistics 2018: GLOBOCAN estimates of incidence and mortality worldwide for 36 cancers in 185 countries. CA Cancer J Clin 68: 394-424.

4. Capote Negrin LG (2015) Epidemiology of cervical cancer in Latin America. Ecancermedicalscience.

5. WHO | Human papillomavirus (HPV).

6. Vallès $X$, Murga GB, Hernández $G$, Sabidó $M$, Chuy $A$, et al. (2009) High prevalence of human papillomavirus infection in the female population of Guatemala. Int $\mathrm{J}$ Cancer 125: 1161-1167.

7. Andersson S, Safari H, Mints M, Lewensohn-Fuchs I, Gyllensten $U$, et al. (2005) Type distribution, viral load and integration status of high-risk human papillomaviruses in pre-stages of cervical cancer (CIN). Br J Cancer 92: 21952200.

8. Cancer today.

9. Murillo R, Herrero R, Sierra MS, Forman D (2016) Cervical cancer in Central and South America: Burden of disease and status of disease control. Cancer Epidemiol 1: S121-S130.

10. Barut MU, Kale A, Kuyumcuoğlu U, Bozkurt M, Ağaçayak E, et al. (2015) Analysis of Sensitivity, Specificity, and Positive and Negative Predictive Values of Smear and Colposcopy in Diagnosis of Premalignant and Malignant Cervical Lesions. Med Sci Monit Int Med J Exp Clin Res 21: 38603867.

11. Petry KU, Schmidt D, Scherbring S, Luyten A, ReineckeLüthge A, et al. (2011) Triaging Pap cytology negative, HPV positive cervical cancer screening results with $\mathrm{p} 16 / \mathrm{Ki}-67$ Dual-stained cytology. Gynecol Oncol 121: 505-509.

12. Agoff SN, Lin P, Morihara J, Mao C, Kiviat NB, et al. (2003) p16(INK4a) expression correlates with degree of cervical neoplasia: a comparison with $\mathrm{Ki}-67$ expression and detection of high-risk HPV types. Mod Pathol Off J U S Can AcadPathol Inc 16: 665-673.

13. Ordi J, Sagasta A, Munmany M, Rodríguez-Carunchio L, Torné A, et al. (2014) Usefulness of p16/Ki67 immunostaining in the triage of women referred to colposcopy. Cancer Cytopathol 122: 227-235.

14. Ikenberg $\mathrm{H}$, Bergeron $\mathrm{C}$, Schmidt D, Griesser $\mathrm{H}$, Alameda F, et al. (2013) Screening for Cervical Cancer Precursors With p16/Ki-67 Dual-Stained Cytology: Results of the PALMS Study. J Natl Cancer Inst 195: 1550-1557.

15. Wentzensen N, Schwartz L, Zuna RE, Smith K, Mathews C, et al. (2012) Performance of $p 16 / K i-67$ immunostaining to detect cervical cancer precursors in a colposcopy referral population. Clin Cancer Res Off J Am Assoc Cancer Res 18: 4154-4162.

16. Bao H, Wu Y (2011) p16INK4A and Ki-67 immunostaining on cell blocks from residual ThinPrep material is helpful in identifying significant preneoplastic cervical lesions. Pathol Res Pract 207: 216-219.

17. Hebbar A, Murthy VS (2017) Role of p16/INK4a and Ki-67 as specific biomarkers for cervical intraepithelial neoplasia: An institutional study. J Lab Physicians 9: 104-110.

18. Wentzensen N, Fetterman B, Tokugawa D, Schiffman M, Castle PE, et al. (2014) Interobserver reproducibility and 
accuracy of p16/Ki-67 dual-stain cytology in cervical cancer screening. Cancer Cytopathol 122: 914-920.

19. Bergeron C, Ordi J, Schmidt D, Trunk MJ, Keller T, et al. (2010) Conjunctive p16INK4a testing significantly increases accuracy in diagnosing high-grade cervical intraepithelial neoplasia. Am J Clin Pathol 133: 395-406.

20. Lee KR, Ashfaq R, Birdsong GG, Corkill ME, McIntosh KM, et al. (1997) Comparison of conventional Papanicolaou smears and a fluid-based, thin-layer system for cervical cancer screening. Obstet Gynecol 90: 278-284.

21. Murphy N, Ring M, Killalea AG, Uhlmann V, O'Donovan M, et al. (2003) p16INK4A as a marker for cervical dyskaryosis: CIN and cGIN in cervical biopsies and ThinPrepTM smears. J Clin Pathol 56: 56-63. 